POS $\quad$ PROCEEDINGS

\title{
Centre vortices and the quark propagator
}

\section{Patrick O. Bowman*}

Institute of Fundamental Sciences and Centre for Theoretical Chemistry and Physics, Massey

University, Albany, New Zealand

E-mail: p.o.bowman@massey.ac.nz

\section{Kurt Langfeld}

School of Maths \& Stats, University of Plymouth, Plymouth, England

\section{Alan O'Cais, Derek B. Leinweber, André Sternbeck, Lorenz von Smekal and Anthony G. Williams \\ Centre for the Subatomic Structure of Matter (CSSM), School of Chemistry \& Physics, University of Adelaide, Australia}

\section{Daniel Jens-Kusterer}

Institut für Theoretische Physik, Universtät Tübingen, Germany

\begin{abstract}
It is thought that confinement and chiral symmetry breaking might be driven by the same mechanism. Centre vortices have long been considered a promising candidate for such a mechanism. We use the Landau-gauge quark propagator as a probe of dynamical chiral symmetry breaking and show that, for SU(2) gauge theory, the infrared behaviour of the quark propagator is indeed dominated by the vortex matter. This is in constrast to the $\mathrm{SU}(3)$ theory, where $\mathrm{D} \chi \mathrm{SB}$ isseen to survive even on non-confining, vortex-removed configurations.
\end{abstract}

8th Conference Quark Confinement and the Hadron Spectrum September 1-6, 2008

Mainz. Germany

\footnotetext{
* Speaker.
} 


\section{Introduction}

Dynamical breaking of chiral symmetry $(\mathrm{D} \chi \mathrm{SB})$ is an essential nonperturbative property of Quantum Chromodynamics (QCD) absent from any order of perturbation theory. The other characteristic nonperturbative phenomenon of QCD is confinement: the fact that coloured states are never observed. It is tempting to speculate that these two phenomena might be driven by a single mechanism, an idea supported by finite-temperature studies where the deconfinement and chiral restoration transitions are observed to occur at coincident temperatures [1]. Moreover, it was found that the low-lying modes of the quark operator not only bear witness to $\mathrm{D} \chi \mathrm{SB}$ but also to confinement [2]. One leading candidate for such a mechanism is the centre vortex. The recovery of the string tension from "vortex-only" $\mathrm{SU}(2)$ gauge configurations (i.e., $\mathrm{Z}_{2}$ projected from $\mathrm{SU}(2)$ ) is well known, as is the recovery of the chiral condensate [3-5]. We use the Landau-gauge quark propagator as a probe of $\mathrm{D} \chi \mathrm{SB}$. The Dirac scalar part of the propagator, related at large momenta to the perturbative running mass, is enhanced at low momenta, even in the chiral limit [6]: a demonstration of $\mathrm{D} \chi \mathrm{SB}$. We explicitly establish in $\mathrm{SU}(2)$ the relation between centre vortices and $\mathrm{D} \chi \mathrm{SB}$ by investigating the quark propagator under the removal of centre vortices. Specifically, we will provide numerical evidence that dynamical mass generation disappears if those vortices are removed, and surprisingly, much of it resides in the vortex-only part. In SU(3), however, the situation is far less compelling. There, mass generation remains intact even after removing centre vortices, while the string tension vanishes as expected.

\section{Details of the calculation}

$\mathrm{SU}(2)$ configurations were generated on a $16^{3} \times 32$ lattice using a tadpole-improved Wilson gauge action with an inverse coupling constant $\beta=1.35$. Around 120 configurations were used. $100,16^{3} \times 32, \mathrm{SU}(3)$ configurations were generated using a Symanzik improved action gauge action at $\beta=4.60$.

\subsection{Identifying centre vortices}

The observation that the long-range static potential only depends on the centre charge (also called "N-ality") of the quark representation led to the expectation that the centre subgroup $Z_{N}$ of $S U(N)$ plays a crucial role for quark confinement. Early attempts to define this subgroup by the projection $S U(N) \rightarrow Z_{N}$ failed in the sense that the arising vortex matter did not have meaningful properties in the continuum limit. A two step process is generally used [7] to define the links $Z_{\mu}(x)$ spanning the $Z_{N}$ gauge theory:

$$
\begin{aligned}
& \text { (i) } \quad \sum_{x, \mu}\left|\operatorname{Tr} U_{\mu}^{g}(x)\right|^{2} \stackrel{g}{\longrightarrow} \max \\
& \text { (ii) } \quad \Re \operatorname{Tr}\left(U_{\mu}^{g}(x) Z_{\mu}^{\dagger}(x)\right) \stackrel{g}{\longrightarrow} \max .
\end{aligned}
$$

Using a standard iterative overrelaxation procedure for step (i) defines vortex matter with a sensible phenomenology in the continuum limit: for $\mathrm{SU}(2)$, it was observed that the so-called "vortexonly" configurations, defined by the centre links $Z_{2}(x)$, reproduce the string tension while "vortex- 
removed" configurations, spanned by the links

$$
\widetilde{U}_{\mu}(x)=Z_{\mu}^{\dagger}(x) U_{\mu}(x),
$$

do not support a linear rising static potential at large distances [7].

Unfortunately, vortex properties do depend on the set of maxima of Eq. $(2.1)[8,9]$. Localising the global maximum of Eq. (2.1) would remove this ambiguity, but the vortex matter arising might not be of any phenomenological value: the larger the gaugefixing functional Eq. (2.1), the less the string tension obtained from vortex-only configurations $[9,10]$. Although a concise mathematical description is yet to be found, the vortex matter best for phenomenological studies seems to arise from an average over Gribov copies.

For SU(2) we employ a novel gaugefixing method [11], introducing an aspect of simulated annealing. We calculated the planar vortex area density in units of the (measured) string tension for several values of the lattice spacing. The vortex density becomes independent of the lattice regulator for sufficiently small values of the lattice spacing. For SU(3) there is a number of possible gaugefixing choices. The gaugefixing functional of Eq. (2.1) corresponds to the so-called "mesonic" gauge condition [4], which is employed in this studied. Standard methods are used to fix the configurations to Landau gauge. The gauge potential has the usual linear definition.

\subsection{The AsqTad quark propagator}

In covariant gauges in Euclidean momentum space the quark propagator can be parametrized by the general form

$$
S\left(p^{2}\right)=\frac{Z\left(p^{2}\right)}{i \gamma \cdot p+M\left(p^{2}\right)}
$$

where $M$ is the running quark mass and $Z$ the quark dressing function. $S$ can be calculated in a lattice regularization where the lattice spacing, $a$, makes all expressions finite. At sufficiently small $a$, i.e., if scaling violations due to finite lattice spacings are negligible then the bare quark propagator, $S$, is related to the renormalised propagator via multiplicative renormalisation. In MOM schemes $S$ is required have the form of a free propagator at the renormalisation point $p^{2}=\mu^{2}$. The mass function, $M$, is renormalisation group invariant.

For the gauge we used Landau gauge: it is straightforward to implement on the lattice and allows for an easy comparison to other studies. The Landau-gauge quark propagator has been studied widely in SU(3) gauge theory using a variety of quark actions in quenched and unquenched simulations (see, e.g, Refs. $[6,12,13]$ and references therein). It has been shown that the quark propagator obtained with the AsqTad improved staggered quark action possesses good symmetry properties and is well-behaved at large momenta. The AsqTad action is therefore a natural choice for this study.

We define a "kinematic momentum" from the tree-level form of the lattice AsqTad quark propagator [6]. This, known as tree-level correction [14], accounts for the lowest order breaking of rotational symmetry. We assume that with this definition of momentum the continuum decomposition of the quark propagator is a good approximation of that for the lattice quark propagator. A cylinder cut [14] is applied to all the data to eliminate points most contaminated by the effects of rotational symmetry violation. 

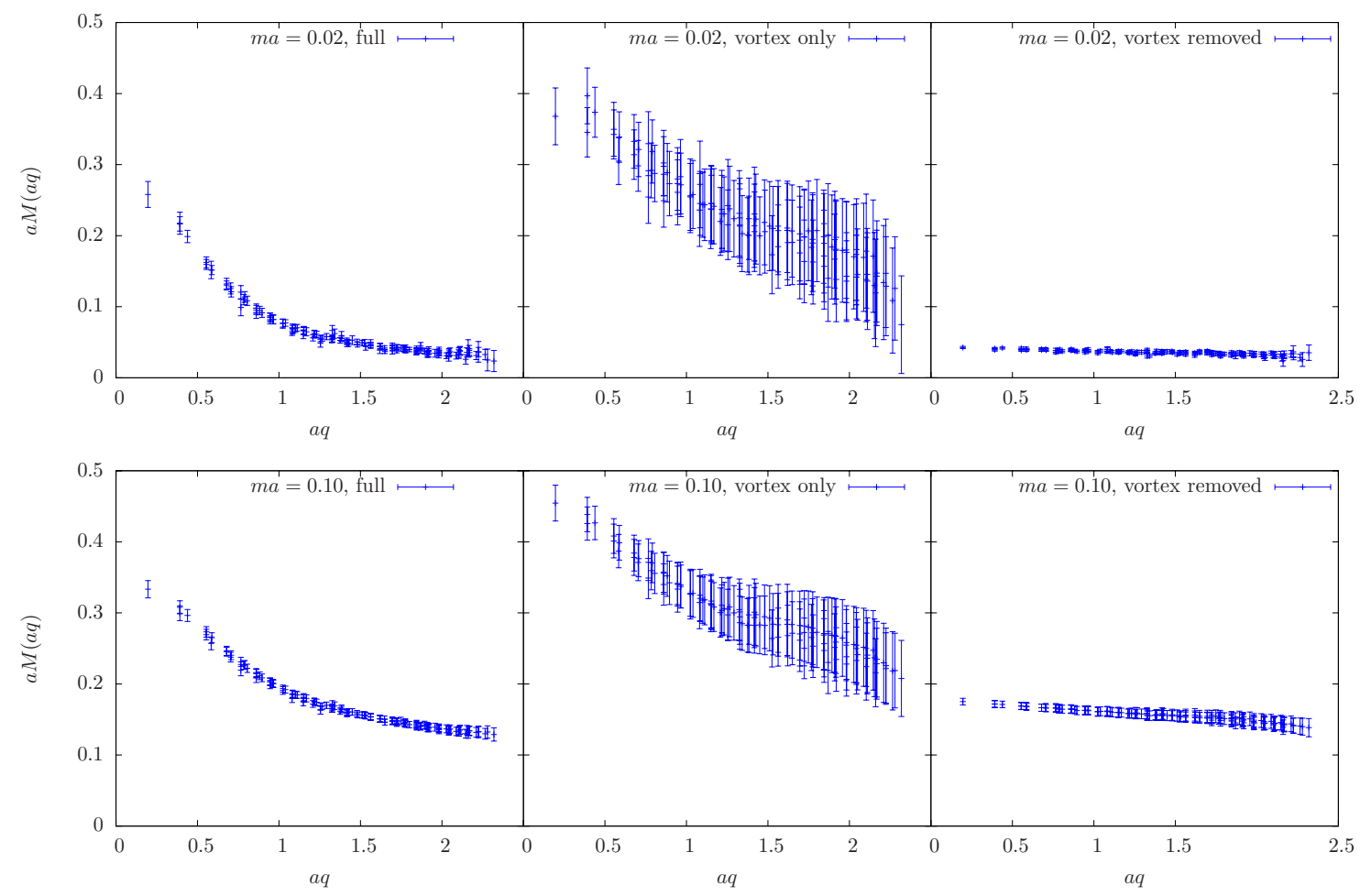

Figure 1: The mass function $a M(q)$ versus kinematic momentum for two bare quark masses $m a=0.02$ (top) and $m a=0.10$ (bottom). We show data on full, vortex-only and vortex-removed configurations from left to right. Data has been cylinder cut. The nonperturbative enhancement of the mass function at low momenta is associated with the presence of centre vortices.

\section{Results}

\subsection{SU(2)}

In Fig. 1 we compare the mass function on our sets of full, vortex-only and vortex-removed configurations. Data was obtained for a range of bare quark masses from $m a=0.020$ to $m a=$ 0.100 , and those two extremes are shown here as functions of momentum. Our results on the full SU(2) configurations (left column) show a large enhancement near zero momentum, whilst the data points drop rapidly to the expected asymptotic behaviour at large momenta. The infrared enhancement is strongest for the smallest bare quark mass. This is just what one would expect from the many studies of the Landau-gauge quark propagator in SU(3).

On the vortex-removed configurations (Fig. 1 right column), the mass function is more or less flat taking values slightly above $m a$. Thus, the dynamical contribution to the mass function, which we clearly see on the full configurations, disappears when centre vortices are removed. Interestingly, the mass function on the vortex-only configurations (Fig. 1, middle column) depends strongly on momentum, even if the signal is quite noisy. That is, much of the infrared enhancement of $M$ is contained in the vortex-only part, which clearly demonstrates the importance of centre vortices as IR degrees of freedom.

The same comparison was made for the bare quark dressing function, $Z$. On the full config- 



Figure 2: SU(3) quark propagator with (open black circles) and without (solid red squares) centre vortices.

urations, the quark dressing function takes values around one at large momenta and is suppressed at low momentum. The smaller the quark mass the more pronounced the dip at lower momenta. If centre vortices are removed, the infrared suppression disappears and $Z$ is a flat function of momentum, staying approximately at its tree-level value. On the vortex-only configurations, $Z$ has a similar momentum dependence to the full configurations. Again, the results are noisier, but the infrared suppression is unambiguous.

\section{2 $\mathrm{SU}(3)$}

Figure 2 shows the quark mass and renormalisation functions for the SU(3) gauge theory. The results for the full (untouched) configurations are shown in open (black) circles while the solid (red) squares show the functions on configurations where the centre vortices have been removed. A roughening of the mass function at large momenta indicates that the removal of centre vortices introduces significant noise into the gauge field configurations giving rise to a larger effective mass, but the mass function is still strongly momentum dependent. Mass generation remains even after removing the centre vortices. The wavefunction renormalisation function is even less affected by the removal of the centre vortices, just flattening slightly at the lowest momentum points. It is astonishing that the dynamical mass generation survives without the vortices. Note that these are configurations on which the string tension vanishes.

\section{Conclusions}

We have studied the Landau-gauge quark propagator in quenched SU(2) and SU(3) gauge theories under the removal of centre vortices. Using the AsqTad improved staggered quark action, the SU(2) quark propagator was found to strongly resemble that of the SU(3) theory.

Our results for the mass and quark dressing functions unambiguously show, in $\mathrm{SU}(2)$, the disappearance of $\mathrm{D} \chi \mathrm{SB}$ when centre vortices are removed. This is consistent with the vanishing of the chiral condensate under similar conditions [3]. In contrast, in SU(3) dynamical mass generation survives after centre vortex removal, while the static quark potential is flat. Additionally, we have studied the quark propagator on vortex-only configurations. Even though the signal is quite noisy, both parts of the propagator reveal a form qualitatively similar to the full, untouched configurations. Our $\mathrm{SU}(2)$ results clearly represent a strong relationship between the vortex picture and 
spontaneous breaking of chiral symmetry. In SU(3), the vortex-only results were even noisier than the $S U(2)$ results, making it difficult to draw any conclusions from them.

In $\mathrm{SU}(3)$ the identification of the vortices is complicated by the fact that $Z_{3}$ has two elements, which means that there are two interacting species of vortex. What rôle this plays in the failure of the current implementation of the centre vortex model remains for future investigation.

\section{ACKNOWLEDGMENTS}

This research made use of the publicly available MILC code, and was supported by the Australian Research Council, eResearch South Australia, the Australian Partnership for Advanced Computing and the Marsden Fund of the Royal Society of New Zealand.

\section{References}

[1] E. Laermann and O. Philipsen, Status of lattice QCD at finite temperature, Ann. Rev. Nucl. Part. Sci. 53, 163 (2003), hep-ph/0303042.

[2] F. Synatschke, A. Wipf, and K. Langfeld, Relation between chiral symmetry breaking and confinement in YM-theories, Phys. Rev. D77, 114018 (2008), 0803.0271.

[3] P. de Forcrand and M. D'Elia, On the relevance of center vortices to QCD, Phys. Rev. Lett. 82, 4582 (1999), hep-lat/9901020.

[4] K. Langfeld, Vortex matter in SU(3) lattice gauge theory, Phys. Rev. D69, 014503 (2004), hep-lat/0307030.

[5] J. Gattnar et al., Center vortices and Dirac eigenmodes in SU(2) lattice gauge theory, Nucl. Phys. B716, 105 (2005), hep-lat/0412032.

[6] P. O. Bowman, U. M. Heller, D. B. Leinweber, A. G. Williams, and J. B. Zhang, Quark propagator from LQCD and its physical implications, Lect. Notes Phys. 663, 17 (2005).

[7] L. Del Debbio, M. Faber, J. Giedt, J. Greensite, and S. Olejnik, Detection of center vortices in the lattice Yang-Mills vacuum, Phys. Rev. D58, 094501 (1998), hep-lat/9801027.

[8] T. G. Kovacs and E. T. Tomboulis, On P-vortices and the Gribov problem, Phys. Lett. B463, 104 (1999), hep-lat/9905029.

[9] V. G. Bornyakov, D. A. Komarov, and M. I. Polikarpov, P-vortices and drama of Gribov copies, Phys. Lett. B497, 151 (2001), hep-lat/0009035.

[10] A. O. Cais et al., Preconditioning Maximal Center Gauge with Stout Link Smearing in SU(3), (2008), 0807.0264.

[11] P. O. Bowman et al., Center vortices and the quark propagator in $S U(2)$ gauge theory, Phys. Rev. D78, 054509 (2008), 0806.4219.

[12] M. B. Parappilly et al., Scaling behavior of quark propagator in full QCD, Phys. Rev. D73, 054504 (2006), hep-lat/0511007.

[13] W. Kamleh, P. O. Bowman, D. B. Leinweber, A. G. Williams, and J. Zhang, Unquenching effects in the quark and gluon propagator, Phys. Rev. D76, 094501 (2007), arXiv:0705.4129 [hep-lat].

[14] UKQCD, D. B. Leinweber, J. I. Skullerud, A. G. Williams, and C. Parrinello, Asymptotic scaling and infrared behavior of the gluon propagator, Phys. Rev. D60, 094507 (1999), hep-lat/9811027. 